\title{
Manifestations buccales de l'infection virale C chronique
}

\author{
Oral manifestations of chronic hepatitis C infection
}

FOUZIA HAKKOU, SALIHA CHBICHEB, WAFAE EL WADI

\section{RÉSUMÉ}

Depuis sa découverte en 1989 et la mise au point de techniques de dépistage de l'infection en 1990, le virus de l'hépatite $\mathrm{C}(\mathrm{VHC})$ a pris une place très importante en hépato-gastro-entérologie, - il est à l'origine de la plupart des hépatopathies chroniques - mais aussi dans la plupart des spécialités médicales car l'infection chronique par le VHC peut s'accompagner de très nombreuses atteintes extrahépatiques. Parmi ces dernières, beaucoup ne sont que des cas rapportés ou ne sont pas étayés par des preuves suffisantes. D'autres, en revanche, semblent clairement associés au VHC même si les mécanismes physiopathologiques ne sont pas tous élucidés. Ce travail rapporte les manifestations extrahépatiques, liées au VHC, les plus fréquemment rapportés dans la littérature et qui impliquent principalement ou exclusivement la région buccale. (Med Buccale Chir Buccale 2009 ; 15: 31-37).

mots clés: hépatite $C$, syndrome de Gougerot-Sjögren, lichen plan buccal, purpura thrombopénique autoimmun

\section{SUMMARY}

Since its discovery in 1989 and the development of techniques for detection of infection in 1990, the hepatitis C virus (HCV) took a very important place, not only in hepato-gastroenterology - since it is the source of most of the chronic liver diseases - but also in all medical specialties because some widespread extrahepatics have been linked to chronic infection with HCV. Among these, much are only reported cases or are not supported by reasonable evidence. Others, however, seem clearly associated with HCV even if the physiopathological mechanisms are not all clarified. This article reports the events extrahepatic, related to the $\mathrm{VHC}$, the most frequently reported by the literature and which involve predominantly or exclusively the oral region. (Med Buccale Chir Buccale 2009 ; 15: 31-37).

key words: hepatitis C, Sjögren syndrome, oral lichen planus, autoimmune thrombopenia pupura 
médecine

buccale

chirurgie

buccale

VOL. $15, \mathrm{~N}^{\circ} 1$ 2009

page 32
L'hépatite $\mathrm{C}$ est un problème majeur de santé à l'échelle du monde. L'Organisation Mondiale de la Santé (OMS) estime que 170 millions de personnes dans le monde sont des porteurs chroniques du $\mathrm{VHC}$ ( $3 \%$ de la population mondiale), et que 3 à 4 millions d'individus sont infectés chaque année par ce virus, donc exposés au risque de cirrhose et de cancer du foie.

Le virus de l'hépatite $\mathrm{C}(\mathrm{VHC})$ est un virus à $A R N$ appartenant à la famille des Flaviviridae. II présente une hétérogénéité génétique importante due au taux élevé de mutations lors de la réplication. Cette variabilité génomique a permis de distinguer différents types, sous types et quasiespèces du VHC.

Transmis principalement par le sang, le VHC s'est répandu de manière relativement silencieuse par transfusions sanguines pendant plusieurs décennies. II s'est aussi répandu massivement dans la population des toxicomanes utilisant la voie intraveineuse, et il est maintenant reconnu comme un virus à transmission nosocomiale [1].

L'implication de l'infection par le VHC a été suspectée, puis démontrée dans de nombreuses pathologies, secondairement dénommées manifestations extrahépatiques (MEH) liées au VHC. Parmi ces dernières, beaucoup ne sont que des cas rapportés ou ne sont pas étayées par des preuves suffisantes. D'autres, en revanche, comme les cryoglobulinémies mixtes et leurs manifestations cliniques, et certains types de lymphomes semblent clairement associées au VHC, même si les mécanismes physiopathologiques ne sont pas tous élucidés. [2]

Les manifestations buccales de l'infection chronique virale $C$ les plus fréquemment rapportés par la littérature sont le syndrome sec, le syndrome de Gougerot-Sjögren (SGS) et le lichen plan buccal.

\section{SYNDROME SEC ET SYNDROME DE GOUGEROT-SJÖGREN (SGS) (Fig.1)}

Plusieurs éléments ont initialement suggéré l'existence de liens entre le SGS et l'infection par le VHC : association fréquente entre cryoglobulinémies mixtes (CM) et VHC, prévalence élevée du SGS au cours des CM essentielles, fréquence de

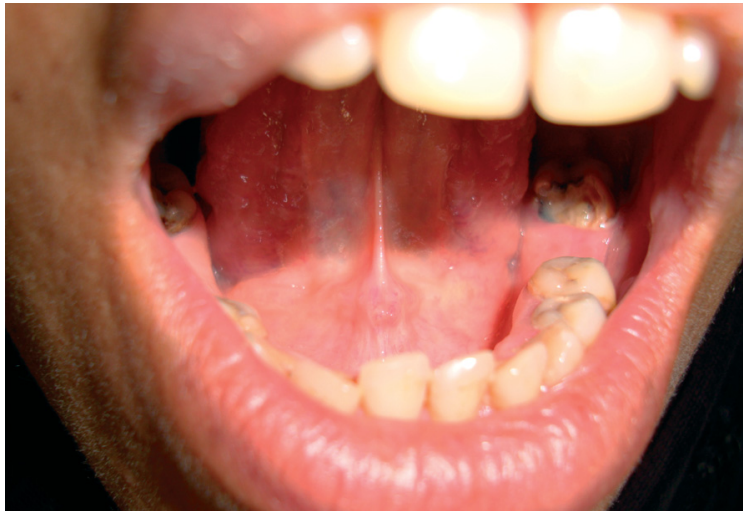

Figure 1: Absence de salive sur le plancher buccal dans un syndrome de Gougerot-Sjögren.

Lack of saliva usually present in the mouth floor in a Sjögren's syndrome.

la détection du VHC dans la salive $(50 \%$ des malades). Ces hypothèses ont semblé trouver une confirmation dans l'étude de Haddad et al. ${ }^{[3]}$ qui rapportait une sialadénite lymphocytaire chez $57 \%$ des malades infectés par le VHC contre seulement $5 \%$ d'un groupe de sujets témoins. Toutefois, dans cette série, $1 / 3$ des malades seulement avait une xérostomie, aucun n'avait de xérophtalmie et aucun n'avait les auto-anticorps habituellement trouvés au cours du SGS (antiSSA, anti-SSB).

De nombreuses études prospectives ultérieures ont permis de mieux définir les liens entre VHC, syndrome sec et SGS [4,5]. Dans les cohortes de malades présentant un SGS primitif confirmé (xérostomie, xérophtalmie, classe III et IV de Chisholm sur la biopsie des glandes salivaires, présence d'anticorps anti-SSA ou anti-SSB), les anticorps anti-VHC n'étaient présents que chez 4 à $11 \%$ des malades ; une seule étude trouvant un pourcentage plus élevé de l'ordre de $19 \%$ [6]. Dans les études prospectives de patients chroniquement infectés par le VHC, la prévalence du syndrome sec buccal et/ou oculaire est de 9 à $67 \%$. Les écarts importants de ces prévalences sont essentiellement liés aux critères très variables utilisés pour la définition du SGS [2]. Ainsi, dans l'étude de Loustaud-Ratti et al. [7] qui inclut 45 patients infectés par le VHC, la prévalence du SGS variait de manière significative selon les critères diagnostiques : critères européens 24 patients (53\%), critères de Manthorpe 25 patients $(56 \%)$ et critères de Fox quatre 
patients (9 \%). Globalement, 11 à $13 \%$ des patients présentent une xérostomie et/ou une xérophtalmie.

Sur le plan clinique, les études font des descriptions comparables des patients ayant un SGS primitif et un SGS secondaire [7]. Une étude multicentrique réalisée par le "SGS-HCV Study Group » a comparé 137 patients infectés par le VHC et porteurs d'un SGS à 400 patients atteints d'un SGS primitif [8]. Ce travail permet de préciser les caractéristiques du SGS associé au VHC ; les manifestations glandulaires sont aussi fréquentes dans les deux groupes mais les signes cliniques de vascularites et la présence de neuropathies périphériques sont plus souvent observés chez les patients porteurs du VHC. Le profil immunologique du SGS associé au VHC est caractérisé par la fréquence des cryoglobulinémies (50\%), de l'hypocomplémentémie et par la rareté des anticorps anti-SSA ou anti-SSB ${ }^{[8]}$. Les membres du «SGS-HCV Study Group » considèrent que l'infection par le VHC doit être un critère d'exclusion du SGS primitif, mais proposent d'inclure le VHC dans les causes de SGS secondaires $[8,9]$.

L'examen histologique des glandes salivaires de patients infectés par le VHC objective en général des lésions très différentes de celles retrouvées au cours du SGS : infiltrat cellulaire péricapillaire et non péricanalaire, importance de l'infiltrat lymphocytaire et absence de destruction des canaux glandulaires. La sialadénite lymphocytaire a également été rapportée au cours d'autres infections virales ou d'autres maladies chroniques du foie non virales.

Sur le plan physiopathologique, le mécanisme lésionnel entraînant le syndrome sec chez des patients infectés par le VHC semble passer par la présence du virus lui-même dans les glandes salivaires et/ou lacrymales. Dans un modèle de souris transgénique, l'hyperexpression des protéines E1 et E2 du VHC, entraîne le développement d'une sialadénite assez proche de celle observée chez les patients. Un mécanisme physiopathologique récemment proposé résulterait d'un mimétisme moléculaire, les anticorps antigpE2 réagissant contre une protéine cytosolique des cellules épithéliales des glandes salivaires [2]. Une étude a évalué la prise en charge thérapeu- tique du SGS associé au VHC. Dans une petite série de 12 patients, Doffoël-Hantz et al. [10] ont apprécié l'effet de I'INF $\alpha$ d'une part, et de l'association INF $\alpha$-ribavirine d'autre part, chez ces patients. Le syndrome sec n'a été amélioré que chez la moitié d'entre eux et uniquement avec la bithérapie. Plusieurs patients ont présenté une complication immunologique sévère sous traitement par l'INF $\alpha$.

Au total, si un syndrome sec buccal et/ou oculaire est fréquemment présent chez les patients chroniquement infectés par le VHC, l'association VHC-SGS est beaucoup plus rare.

\section{LICHEN PLAN BUCCAL (LPB) (Fig.2)}

Le lichen plan est une dermatose inflammatoire chronique, généralement bénigne, caractérisée par un trouble de kératinisation et dont les aspects cliniques sont polymorphes. II peut atteindre la peau, les phanères, les muqueuses malpighiennes (muqueuse buccale surtout), et plus rarement d'autres muqueuses (génitale, œsophage). Sur le plan histopahtologique, il est caractérisé par un infiltrat dermique lymphocytaire assez caractéristique ${ }^{[11,12]}$. Sur le plan biologique, des perturbations des fonctions hépatiques sont retrouvées dans environ $20 \%$ des cas de lichen plan pour certains auteurs [13].

L'étiopathogénie du LPB reste inconnue et de nombreux facteurs sont incriminés. Mais la tendance est de considérer le lichen plan comme une

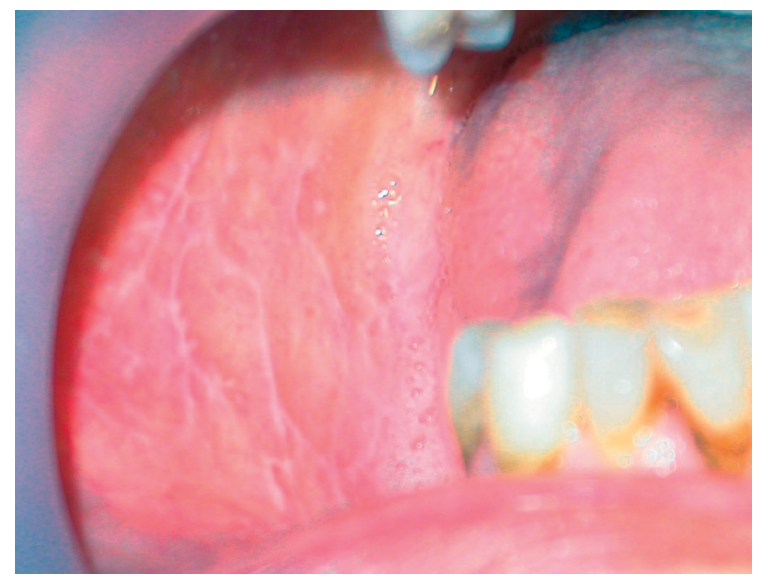

Figure 2 : Lichen plan réticulé sur la muqueuse jugale. Reticular lichen planus on the buccal mucosa. médecine

buccale

chirurgie

buccale

VOL. $15, \mathrm{~N}^{\circ} 1$ 2009

page 33 
médecine buccale chirurgie buccale

VOL. $15, \mathrm{~N}^{\circ} 1$ 2009 page 34 dermatose d'origine immunologique, et peut-être même auto-immune. Cette hypothèse autoimmune repose sur l'observation fréquente d'une association entre LPB et certaines maladies autoimmunes (réaction du greffon contre l'hôte, néoplasies, pemphigus, lupus érythémateux). On connait également l'association LPB et certaines hépatopathies, principalement l'hépatite virale $\mathrm{C}$. Sur le plan épidémiologique, la fréquence de la co-affection VHC-LPB change considérablement : en effet, la prévalence des anticorps anti-VHC chez les sujets présentant un LPB varie de $0 \%$ en Ecosse et en Hollande à $62 \%$ au Japon (Tab. 1). Tandis que la prédominance du LPB chez les patients infectés par le VHC varie de $1.6 \%$ en Italie à $20 \%$ en Ecosse (Tab. 2). Toutefois, il convient de noter que peu d'études ont porté sur la prédominance du LPB chez les patients atteints d'hépatite $C$, comparé à celles qui ont évalué la prédominance du VHC chez les patients présentant un LPB.
Sur le plan physiopathologique, plusieurs publications suggèrent une association significative entre le LPB et le VHC sans pouvoir apporter une explication physiopathologique satisfaisante. Les deux hypothèses principales expliquant le rôle du VHC dans la genèse de LPB sont les suivantes: - La réplication de $\mathrm{VHC}$ dans l'épithélium buccal contribuerait directement au développement des lésions de LPB.

- Le taux élevé de mutation du VHC aurait comme conséquence l'activation répétée des cellules immunitaires augmentant ainsi la probabilité de réactions croisées dans les tissus buccaux, favorisant ainsi le risque de développement des maladies auto-immunes.

- L'origine géographique et la prédisposition génétique semblent jouer un rôle car la prévalence de l'association LPB - HVC varie d'une région géographique à l'autre ${ }^{[25]}$. Cela peut être expliqué par les différences dans les facteurs

Tableau 1 : Etudes de la prévalence de l'infection par le VHC chez les patients présentant un LPB. Studies of the prevalence of HCV infection in patients with LPB .

\begin{tabular}{|l|c|c|}
\hline \multicolumn{1}{|c|}{ Etude } & Origine & $\begin{array}{c}\text { Prévalence du VHC chez les patients } \\
\text { présentant un LPB }\end{array}$ \\
\hline Chainani-Wu et coll. 2001 [14] & Californie & $14 / 31(45 \%)$ \\
\hline Tanei et coll. 1995 [15] & Japon & $17 / 45$, de tous les types de LP (37,8\%) \\
\hline Dupond et coll. 1998 [16] & France & $8 / 28(29 \%)$ \\
\hline Nagae et coll. 1995 [17] & Japon & $28 / 45(62 \%)$ \\
\hline Rossi et Colasanto 2000 [18] & Italie & $13 / 100(13 \%)$ \\
\hline Roy et coll. 2000 [19] & Ecosse & $0 / 6(0 \%)$ \\
\hline Van der Meij and Van der waal 2000 [20] & Hollande & $0 / 55(0 \%)$ \\
\hline Grote et coll. 1999 [21] & Allemagne & $1 / 24(4,2 \%)$ \\
\hline
\end{tabular}

Tableau 2 : Etudes de la prévalence de LPB chez les patients infectés par le VHC. Studies of the prevalence of LPB in patients with HCV.

\begin{tabular}{|l|c|c|}
\hline \multicolumn{1}{|c|}{ Etude } & Origine & $\begin{array}{c}\text { Prévalence de LPB chez les patients } \\
\text { infectés par VHC }\end{array}$ \\
\hline Henderson et coll. 2001 [2] & Ecosse & $8 / 40(20 \%)$ \\
\hline Grote et coll. 1999 [21] & Allemagne & $3 / 127(2,4 \%)$ \\
\hline Nagae et coll. 2002 [23] & Japon & $5 / 59(8,5 \%)$ \\
\hline Mignogna et coll. 2001 [24] & Italie & $5 / 300(1,6 \%)$ \\
\hline
\end{tabular}


génétiques qui contrôlent la réponse immunitaire de l'hôte [26]. Mais aussi par la variabilité génétique de VHC. C'est ainsi que de nombreuses recherches ont été menées dans ce sens ${ }^{[26]}$ et toutes ont conclu qu'il n'y a pas de différence statistiquement significative entre les différents génotypes du VHC et l'association au LPB [25].

L'INF $\alpha$ représente le traitement de choix de l'hépatite virale $C$. Son action sur le LPB est très controversée et, selon les cas, on peut constater une régression des lésions du LPB, voire une disparition, ou une aggravation. L'INF $\alpha$ pourrait même induire une LPB.

S'il y a une association entre LPB et hépatite virale $C$, il serait intéressant d'identifier chez les patients ayant un LPB un sous-groupe de patients qui seraient plus probablement atteints par le VHC, ceci afin de limiter l'usage de test de dépistage de VHC. Plusieurs auteurs ont essayé d'identifier certaines caractéristiques de LPB chez les malades infectés par le VHC. Là aussi, les données épidémiologiques sont controversées. II semble que les formes cliniques les plus fréquemment associées à l'hépatite virale $\mathrm{C}$ soit la forme érosive et la forme réticulée selon les études [11].

\section{PURPURA THROMBOPENIQUE AUTO-IMMUN (Fig. 3a et b)}

Le purpura thrombopénique auto-immune (PTAl) est défini par une thrombopénie aiguë ou chronique, associée à la présence dans le sérum d'anticorps anti-plaquettes et à l'absence d'une autre étiologie susceptible d'expliquer la thrombopénie, en particulier un hypersplénisme. Le PTAI peut être idiopathique ou associé à une maladie virale, une hémopathie, la prise d'un médicament ou une allo-immunisation. De nombreuses infections virales ont été décrites en association avec le PTAI [27]. La présence fréquente d'une thrombopénie chez les malades infectés chroniquement par le VHC peut relever de plusieurs mécanismes : atteinte périphérique (hypersplénisme, thrombopénie auto-immune par anticorps anti-plaquettes, anticorps anti-phospholipides, ARN du VHC dans les plaquettes) ou atteinte centrale (ARN du VHC

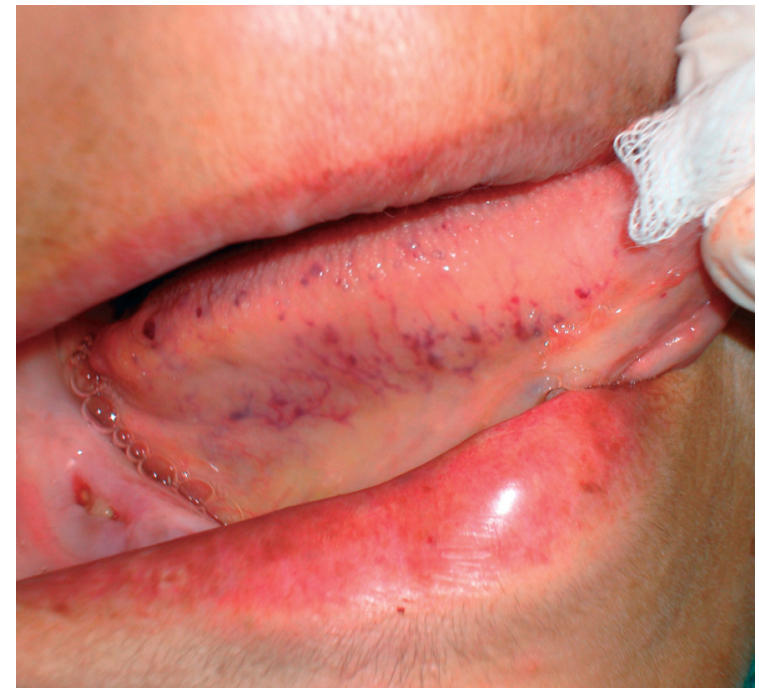

Figure 3a: Purpura de la langue secondaire à une thrombopénie idiopathique.

Purpura of the tongue in idiopathic thrombopenia.

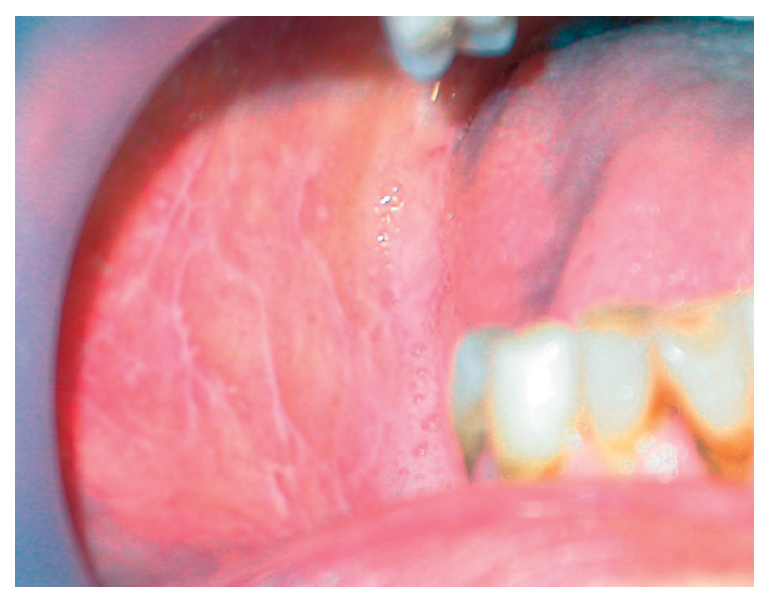

médecine

buccale

chirurgie

buccale

VOL. $15, \mathrm{~N}^{\circ} 1$

2009

page 35
Figure 3b : Ecchymose sur le bord de la langue due à une thrombopénie idiopathique.

Ecchymosis of the tongue in idiopathic thrombopenia.

dans les mégacaryocytes, lymphoprolifération maligne).

Chez les patients atteints d'hépatite chronique C, une thrombopénie modérée est présente dans $41 \%$ des cas. Chez les patients atteints de purpura thrombopénique idiopathique, la prévalence de l'infection virale $C$ varie entre 10 et $25 \%$ [1]. La présence d'anticorps anti-plaquettes était positive chez $88 \%$ des malades atteints d'hépatite C chronique ayant une thrombopénie, et l'ARN du VHC était présent dans les plaquettes 8 fois sur 10. L'effet favorable du traitement anti-VHC, IFN $\alpha$ 
et/ou ribavirine, dans certaines thrombopénies auto-immunes résistantes aux traitements habituels (corticoïdes, disulone, dapsone, immunoglobulines intraveineuses, immunosuppresseurs) renforce l'hypothèse d'un lien réel entre thrombopénie auto-immune et infection par le $\mathrm{VHC}{ }^{[6]}$.

\section{AUTRES MANIFESTATIONS}

De nombreuses autres manifestations extrahépatiques impliquant la région buccale ont été rapportées dans la littérature telles un pemphigus [28,29], une maladie de Behçet ${ }^{[30,31]}$ et un carcinome buccal [32] mais aucune de ces publications ne permet d'établir avec certitude le rôle éventuel du VHC. médecine buccale chirurgie buccale

VOL. $15, N^{\circ} 1$ 2009 page 36

\section{RÉFÉRENCES}

1 - Loustaud-Ratti V, Lunel F. Manifestations extrahépatiques des infections par le virus de l'hépatite C. Rev Prat $2000 ; 50: 1089-92$

2 - Sene D, Saadoun D, Limal N, Piette J-C, Cacoub P. Actualités des manifestations extrahépatiques associées au virus de l'hépatite C. Rev Med Int 2007 ; 28 : 388-93.

3 - Haddad J, Deny P, Munz-Gotheil C, Ambrosini JC, Trinchet JC, Pateron D, Mal F, Callard P, Beaugrand M. Lymphocytic sialadenitis of Sjögren's syndrome associated with chronic hepatitis $\mathrm{C}$ virus liver disease. Lancet 1992 ; 339 : 321-3.

4 - Jorgensen C, Legouffe MC, Perney P, Coste J, Tissot B, Segarra C, Bologna C, Bourrat L, Combe B, Blanc F, Sany J. Sicca syndrome associated with hepatitis C virus infection. Arthritis Rheum 1996 ; 43 : 1166-71.

5 - King PD, MC Murray RW, Becherer PR. Sjögren's syndrome without mixed cryoglobulinemia is not associated with hepatitis C virus infection. Am J Gastroenterol 1994 ; 89 : 1047-50.

6 - Cacoub P. Traitement des manifestations extrahépatiques associées au virus de l'hépatite $\mathrm{C}$. Gastroenterol Clin Biol 2002 ; 26 : B210-B219.

7 - Loustaud-Ratti V, Riche A, Liozon E, Labrousse F, Soria P, Rogez S, Babany G, Delaire L, Denis F, Vidal E. Prevalence and characteristics of Sjögren's syndrome or sicca syndrome in chronic hepatitis $\mathrm{C}$ virus infection: a prospective study. J Rheumatol 2001 ; 28 : 2245-51.

8 - Ramos-Casals M, Loustaud-Ratti V, De Vita S, Zeher M, Bosch JA, Toussirot E, Medina F, Rosas J, Anaya JM,

\section{CONCLUSION}

Souvent asymptomatique, l'hépatite virale $\mathrm{C}$ chronique est parfois découverte à l'occasion de très nombreuses manifestations cliniques extrahépatiques qui peuvent être associées à l'infection chronique par le VHC. Toutefois, les liens de causalité entre les manifestations extrahépatiques et le VHC ne sont pas toujours démontrés, plusieurs questions restent en suspens et doivent faire envisager d'autres d'investigations.

Font J; SS-HCV Study Group. Sjögren syndrome associated with hepatitis $C$ virus: a multicenter analysis of 137 cases. Medicine 2005 ; 84 : 81-9.

9 - Lormeau C, Falgarone G, Roulot D, Boissier M-C. Manifestations rhumatologiques de l'infection chronique virale C. Rev Rhumat 2006 ; 73 : 1287-92.

10 - Doffoël-Hantz V, Loustaud-Ratti V, Ramos-Casals M, Alain S, Bezanahary H, Liozon E, Fauchais AL, Vidal E. Évolution des syndromes de Gougerot-Sjögren associés au virus de l'hépatite $\mathrm{C}$ sous interféron et l'association interféron-ribavirine. Rev Méd Int 2005 ; 26 : 88-94.

11 - Ben Yahya I. Lichen plan buccal, signe prédictif d'une hépatite virale C? Act Odont Stomat 2002 ; 220 : 41340.

12 - Dega H, Le Cleach L. Lichen plan et dermatoses lichénoïdes. Encyc Med Chir 1999; 98-525-A-10.

13 - Agbo-Godeau S, Ragot J-P. Leucoplasie et kératose buccales. Act Odonto Stomato 2004 ; 225 : 23-39.

14 - Chainani-Wu N, Silverman S Jr, Lozada-Nur F, Mayer P, Watson JJ. Oral lichen planus: patient profile, disease progression and treatment responses. J Am Dent Assoc $2001 ; 132: 901-9$.

15 - Tanei R, Watanabe K, Nishiyama S. Clinical and histopathologic analysis of the relationship between lichen planus and chronic hepatitis. C J Dermatol 1995 ; 22 : 316-23.

16 - Dupond AS, Lacour JP, Lafont C, Ortonne JP. Prévalence du virus de l'hépatite $C$ dans le lichen érosif buccal. Ann Dermatol Venereol 1998 ; 125 : 676-8. 
17 - Nagao Y, Sata M, Tanikawa K, Itoh K, Kameyama T. Lichen planus and hepatitis $\mathrm{C}$ virus in the Northern Kyushu region of Japon. Eur J Clin Invest 1995 ; 25 : 910-4.

18 - Rossi L, Colasanto S. Clinical considerations and statistic alanalysis on 100 patients with oral lichen planus. Minerva Stomatol $2000 ; 49$ : 393-8.

19 - Roy KM, Dickson EM, Staines KS, Bagg J. Hepatitis $\mathrm{C}$ virus and oral lichen planus/lichenoid reactions: lack of evidence for an association. Clin Lab 2000 ; 46 : 251-4.

20 - Van Der Meij EH, Van Der Waal I. Hepatitis C virus infection and oral lichen planus: a report from the Netherlands. J Oral Pathol Med 2000 ; 29 : 255-8.

21 - Grote M, Reichart PA, Hopf U. Increased occurrence of oral lichen planus in hepatitis $C$ infection. Mund Kiefer Gesichtschir 1999 ; 3 : 30-3.

22 - Henderson L, Muir M, Mills PR, Spence E, Fox R, McCruden EA, Bagg J. Oral health of patients with hepatitis C virus infection: a pilot study. Oral Dis 2001; $7: 271-5$.

23 - Nagao Y, Tanaka J, Nakanishi T, Moriya T, Katayama K, Kumagai J, Komiya Y, Itoh Y, Myoken Y, Fujihara M, Sata M, Yoshizawa $\mathrm{H}$. High incidence of extrahepatic manifestations in an HCV hyperendemic area. Hepatol Res 2002 ; 22 : 27-36.

24 - Mignogna M, Fedele S, Lo Russo L, Ruoppo E, Lo Muzio L. Unexpected low incidence of oral lichen planus in an HCV hyperendemic area of southern Italy. Gastroenterology 2001; 121 : 1528-9.

25 - Chainani-Wu N, Lozada-Nur F, Terrault N. Hepatitis C virus and lichen planus: a review Oral Surg Oral Med Oral Pathol Oral Radiol Endod 2004 ; 98 : 171-83.
26 - Carrozzo M, Francia Di Celle P, Gandolfo S, Carbone M, Conrotto D, Fasano Me, Et AL. Increased frequency of HLA-DR6 allele in Italian patients with hepatitis $\mathrm{C}$ virusassociated oral lichen planus. Br J Dermatol 2001 ; $144:$ : 803-8.

27 - Duclos-Vallee J-C. Traitement des manifestations extrahépatiques associées à l'infection par le virus de l'hépatite C. Gastroenterol Clin Biol 2002 ; 26 : B76-B81.

28 - Marinho RT, Johnson NW, Fatela NM, Serejo FS, Glória H, Raimundo MO, Velosa JF, Ramalho FJ, Moura MC. Oropharyngeal pemphigus in a patient with chronic hepatitis $C$ during interferon alpha-2a therapy. Eur J Gastroenterol Hepatol 2001 ; 13 : 869-72

29 - Nanda M, Nanda A, Al-Sabah H, Dvorak R, Alsaleh QA. Paraneoplastic pemphigus in association with B-cell lymphocytic leukemia and hepatitis C: favorable response to intravenous immunoglobulins and prednisolone. Int J Dermatol 2007; 46 : 767-9.

30 - Munke H, Stockmann F, Ramadori G. Possible association between Behcet's syndrome and chronic hepatitis C virus infection. N Engl J Med 1995 ; 332 : 400-1.

31 - Farajzadeh S, Shakibi Mr, Moghaddam SD, Rahnama Z. Behcet disease: clinical spectrum and association with hepatitis B and C viruses. East Mediterr Health J 2005 ; $11: 68-72$.

32 - Carrozzo M. Oral diseases associated with hepatitis C virus infection. Part 2: lichen planus and other diseases. Oral Dis 2008 ; 14 : 217-28. \begin{tabular}{l}
$\begin{array}{l}\text { médecine } \\
\text { buccale } \\
\text { chirurgie } \\
\text { buccale }\end{array}$ \\
\hline vOL. $15, N^{\circ} 1$ \\
2009 \\
page 37
\end{tabular} 